Palimpsesto Vol. 11, No 18 (enero-junio, 2021): 101-116

Universidad de Santiago de Chile, ISSN 0718-5898

Tatiana Herrera Rodríguez

Universidad Nacional de Colombia

therrerar@unal.edu.co

\title{
Mestizaje, relaciones maritales y discriminación en el análisis de las relaciones interétnicas en Latinoamérica*
}

\author{
Mestizaje, Marital Relations and Discrimination in the Analysis of \\ Inter-Ethnic Relations in Latin America
}

\begin{abstract}
Resumen
Este artículo presenta un balance bibliográfico de algunas de las tendencias en el análisis de las relaciones interétnicas a partir de la revisión de aproximadamente treinta documentos académicos publicados entre 1995 y 2015 en bases de datos indexadas, repositorios universitarios e instituciones académicas, principalmente, de Colombia, Caribe y Latinoamérica. El corpus documental fue sistematizado en un programa informático de análisis de datos cualitativos, mediante la creación de las categorías-códigos que clasificaban a los artículos por temas, países, perspectivas disciplinarias y periodos temporales. Identificamos algunas de las tendencias de estudio de las relaciones interétnicas y las categorizamos en tres temáticas claves: mestizaje, relaciones maritales y discriminación. A partir del balance, proponemos rutas de indagación para comprender las relaciones interétnicas y potenciar su uso como categoría analítica.
\end{abstract}

Palabras claves: discriminación, mestizaje, Latinoamérica, relaciones interétnicas, relaciones maritales.

\begin{abstract}
This article presents a bibliographic balance of some of the trends in the analysis of inter-ethnic relations from the review of approximately thirty academic documents published between 1995 and 2015 in indexed databases, university repositories and academic institutions, mainly from Colombia, Caribbean and Latin America. The documentary corpus was systematized in a qualitative data analysis computer program, through the creation of categories-codes that classified the articles by themes, countries, disciplinary perspectives and time periods. We identify some of the trends in the study of inter-ethnic relations and categorize them into three key themes: miscegenation, marital relationships and

${ }^{*}$ Este es un artículo de revisión producto de mi trabajo en 2016 como Joven Investigadora de Colciencias en el grupo de investigación Igualdad Racial, Diferencia Cultural, Conflictos Ambientales y Racismos en las Américas Negras (IDCARAN) perteneciente al Centro de Estudios Sociales (CES) de la Universidad Nacional de Colombia bajo la coordinación de Claudia Mosquera Rosero-Labbé.
\end{abstract}


discrimination. Based on the balance, we propose routes of inquiry to understand inter-ethnic relations and promote their use as an analytical category.

Keywords: discrimination, inter-ethnic relations, marital relationships, miscegenation, Latin America.

\section{Introducción}

En el marco del proyecto "Retos en los procesos de autoidentificación étnico-racial en municipios con alto mestizaje interétnico y racial con miras al próximo censo nacional de población y de vivienda en el Caribe colombiano" adelantado por el grupo de investigación sobre Igualdad Racial, Diferencia Cultural, Conflictos Ambientales y Racismos en las Américas Negras (IDCARAN) de la Universidad Nacional de Colombia y coordinado por Claudia Mosquera, llevamos a cabo varias actividades, una de éstas fue la elaboración de un estado de la cuestión sobre el concepto de relaciones interétnicas a partir de la revisión de documentos académicos publicados entre 1995 y 2015. Con base en la pregunta general del proyecto por la autoidentificación étnico-racial y el análisis intratextual de entrevistas realizadas a personas con experiencias de discriminación y procesos de autoreconocimiento étnico-racial, consideramos necesario explorar aquellas relaciones, situaciones o escenarios que pueden incidir en la construcción de la identidad étnico-racial.

Partimos entonces del supuesto según el cual los procesos de autoidentificación étnicoracial, esto es, la percepción que tienen las personas de sí mismas en relación con un grupo étnico, se gestan en la relación con otros individuos y grupos con adscripciones étnicas. Es así que en la indagación por los procesos de construcción de identidad, nos preguntamos si ¿existen las relaciones interétnicas?, ¿cómo se definen?, ¿en qué tipo de situaciones o escenarios se gestan estas relaciones?, ¿esta categoría aporta a la comprensión de autoidentificación étnico-racial? Para responder a estas preguntas, elaboramos un balance de trabajos que se aproximan a las relaciones que inciden o que surgen en la construcción de la identidad étnico-racial. Referirnos a relaciones interétnicas en el marco del proyecto fue pertinente para abordar un tema socialmente sensible como lo es la auto-identificación étnico-racial en Colombia, especialmente, cuando instrumentos de organización de la población como el censo nacional participan de la construcción de categorías que incluyen o excluyen a las personas.

Con base en el estado de la cuestión, el objetivo de este artículo es presentar un balance bibliográfico de algunas tendencias en el análisis de las relaciones interétnicas. Cabe aclarar que esta revisión no constituye el marco teórico del proyecto citado sino una de las líneas de trabajo que surgió del objetivo general de la investigación. Por este motivo, no presentamos el marco teórico general del proyecto, en cambio, lo que nos interesa aquí es poner sobre el tapete los resultados del balance bibliográfico, esto es, algunas de las tendencias en los análisis de las relaciones interétnicas. Así mismo, no discutimos todos los textos revisados sino que nos referimos a aquellos que dan cuenta de los temas de análisis que priorizamos, a saber, mestizaje, relaciones maritales y discriminación.

Para demarcar el horizonte analítico de las relaciones interétnicas, en este texto partimos de las definiciones propuestas por Bari (2002) y Gunderman, González y Durston (2014). Bari 
(2002) rescata de la producción antropológica los conceptos de grupo étnico, etnicidad e identidad étnica para descencializar su contenido y problematizar sus alcances. La autora entiende a los grupos étnicos como organizaciones sociales que se vinculan de manera contrastante y contradictoria con una estructura mayor hegemónica. Llama la atención sobre el carácter relacional y no ontológico de la producción identitaria: la identidad es producto de la relación con otros. Remarca entonces el carácter procesual dinámico de la configuración de etnicidad y la concibe como el resultado de una compleja interacción de relaciones étnicas, las cuales están condicionadas a su vez por relaciones de poder: "consideramos que las relaciones interétnicas son producto de situaciones en las que intervienen grupos sociales con razones y propósitos diversos, y relaciones de poder asimétricas" (2002, p.154).

En su estudio sobre el caso aymara chileno, Gunderman, González y Durston (2014) definen las relaciones interétnicas como "tipos específicos de relación social en que los sujetos interactuantes participan de inscripciones sociales y posicionamientos de etnicidad" (p.397) y "tipos de relaciones entre actores que actúan en tanto que sujetos étnicos" (p.399). Los autores afirman que la activación de "lo étnico" en las interacciones sociales se produce cuando existen condiciones para ello, es decir, que no se da en todos los casos y por ello las relaciones interétnicas tienen como supuesto el reconocimiento social del carácter étnico de la relación (Gunderman, González \& Durston 2014, p.406). De esta manera, las relaciones interétnicas evocan una identidad colectiva en situaciones determinadas, por lo que su comprensión debe tener en cuenta a los actores sociales como social e históricamente situados y localizados en estructuras sociales y de poder que establecen parámetros y límites a su accionar étnico.

Con los anteriores aportes conceptuales y para el propósito de este texto, las relaciones interétnicas se entienden como un tipo de interacción social entre sujetos individuales y colectivos con adscripciones étnicas. Estas relaciones están condicionadas por relaciones de poder y se desenvuelven en escenarios políticos, económicos, sociales y culturales, e incluso íntimos, en las que las condiciones permiten a los individuos y grupos posicionar sus intereses.

A continuación, presentamos brevemente la metodología que seguimos para la revisión, organización y análisis de los documentos académicos consultados. Luego, a partir del balance bibliográfico, describimos las principales tendencias en el análisis de las relaciones interétnicas resaltando algunos de los estudios que revisamos en profundidad. Finalmente, señalamos algunos problemas de investigación a partir de los vacíos identificados.

\section{Metodología para la recolección y análisis del corpus sobre relaciones interétnicas}

Para la elaboración del balance bibliográfico, rastreamos, seleccionamos, revisamos, sistematizamos y categorizamos cerca de noventa documentos académicos que abordaban, desde diversas perspectivas y en distintos contextos, las relaciones interétnicas. Incluimos en el corpus de los documentos aquellos producidos en diversas latitudes, principalmente en Colombia, Caribe y Latinoamérica, pero también en Estados Unidos, África y Europa. De este corpus, analizamos en profundidad cerca de cincuenta textos que nos permitieron responder a nuestra pregunta sobre los procesos de construcción de identidad desde la categoría de relaciones interétnicas. En este artículo presentamos treinta de estos textos y resaltamos algunos de los estudios que dan cuenta de los temas clave de análisis y que se refieren a contextos históricos y actuales de Colombia, Caribe y Latinoamérica. 
La búsqueda y selección de los documentos se realizó a partir de la definición de algunos criterios básicos. En primer lugar, recurrimos a las bases de datos de publicaciones indexadas para rastrear los resultados de investigaciones presentados en artículos durante el periodo comprendido entre 1995 y 2015. Este periodo fue seleccionado por el equipo de trabajo luego de la revisión de metodologías para la elaboración de estados del arte, estados de la cuestión y balances bibliográficos que sugerían un rango amplio de revisión de textos y responde a los últimos veinte años de producción académica recientes en relación con el periodo de desarrollo del proyecto, esto es, entre 2015 y 2016. Además de las bases de datos, acudimos a repositorios universitarios para buscar tesis de grado que abordaran el tema. Finalmente, nos ocupamos de algunos libros producidos por autores y autoras que han trabajado en la materia durante el periodo comprendido.

En segundo lugar, el corpus documental fue sistematizado en el programa informático de análisis de datos cualitativos ATLAS.ti, mediante la creación de las categorías-códigos que nos permitieron clasificar los artículos por temas, países, perspectivas disciplinarias y periodos temporales. Identificamos que las relaciones interétnicas se han estudiado principalmente desde la antropología (Bari; 2002; Bartolomé, 2014; Benedetti, 2012; Cunin, 2002; García, 2010; Gómez, 2013; Gunderman, González \& Durston, 2014; Losonczy, 2006; Meza, 2003; Murray, 2010; Ortega, Nissen \& Castillo, 2006; Rodríguez, 2013; Tiapa, 2011; Wade, 1993, 2003) y la historia (Saether, 2005; Salinas 2012), y en menor medida, la etnohistoria (Flagler, 2008; Polo, 2012), la arqueología histórica (Reyes, 2004), la demografía (Velázquez, 2014) y la sociología (Fabbricatore 2011). Igualmente encontramos estudios sobre el periodo de colonización y república en América Latina en los siglos XVI, XVII, XVIII y XIX (Acuña, 2012; Dueñas, 1997b; Guzmán, 2010; Ibarra, 2002; Petruccelli, 2003; Polo, 2012; Saether, 2005; Velázquez, 2014) en relación con el mestizaje y las relaciones maritales, pero al mismo tiempo, un alto número de autoras y autores se concentra en los siglos XX (González, 2006; Losonczy, 2006) y XXI (Rodríguez, 2013) desde una manera crítica frente a las relaciones de desigualdad y discriminación que permean las relaciones interétnicas. Los trabajos con enfoque antropológico se han encargado de discutir con amplitud el concepto de relaciones interétnicas en el marco de investigaciones de corte etnográfico (Bari, 2002; Losonczy, 2006; Rodríguez, 2012; Rodríguez, 2013). El enfoque antropológico también ha estado acompañado del análisis histórico y el uso de herramientas de la historiografía.

Por último, categorizamos los textos de acuerdo con los temas más recurrentes y que nos permitieron organizar las tendencias de análisis así: "mestizaje", "relaciones maritales" y "discriminación". Por supuesto, en el análisis de las relaciones interétnicas estos no son los únicos temas pero quisimos destacarlos por la producción académica existente. En este balance bibliográfico dejamos por fuera importantes y valiosos trabajos que abordan las relaciones interétnicas desde otros temas: análisis históricos sobre la conformación de estados-nación (Valerezo, 2003) y el establecimiento de fronteras territoriales (Cabrera, 2009; Calderón, 2016; Córdoba \& Villar, 2010; Foerster \& Vezub, 2011; Hernández, 2003; Luiz, 2005); el manejo de las tensiones en las relaciones interétnicas mediante hechizos y ceremonias de acercamiento (Biord, 2005; Losonczy \& Rubiano, 2013); la salud de la población, prácticas médicas y curativas (Casali, 2014; Lenaerts, 2011; Schindler \& Faust 2000); los intercambios comerciales entre grupos étnicos (Galarza, 2012); la intermediación cultural (Ratto, 2005); y las estrategias de inserción en la sociedad en escenarios de participación política (Grebe, 2012). 


\section{Mestizaje, relaciones maritales y discriminación en el análisis de las relaciones interétnicas}

Como lo mencionamos, a partir de los textos revisados agrupamos las tendencias de análisis en relación con el mestizaje, relaciones maritales y discriminación. Con el fin de caracterizar el tipo de relaciones interétnicas que se abordaban en los estudios, a continuación resaltamos algunos de los textos que representan aproximaciones a la comprensión de las relaciones interétnicas desde los temas priorizados en este ejercicio de revisión bibliográfica.

\section{Mestizaje}

El mestizaje es una noción elaborada desde las ciencias sociales para explicar el proceso de contacto entre poblaciones durante el periodo colonial de la historia latinoamericana; puede definirse como un proceso originado en la colonia resultado de la mezcla de las "razas" (Acuña, 2012). Encontramos que buena parte de los documentos analizados abordan el mestizaje como resultado de las relaciones interétnicas entre distintas categorías sociales: personas indígenas, mestizas, mulatas, españolas y esclavas. En estos análisis las relaciones interétnicas son entendidas como las relaciones producto de la convivencia cotidiana entre estas diversas categorías.

Las herramientas analíticas y metodológicas de la historia han conducido el estudio de este proceso desde el siglo XVI y sus particularidades locales a lo largo de los siglos subsiguientes. Los trabajos analizados reconstruyen la configuración de las relaciones entre distintos grupos poblacionales categorizados a partir de la variable racial en distintos momentos históricos del periodo colonial (Acuña, 2012; Almario, 2009; Amodio, 1999; Bossert \& Siffredi, 2011; Buscaglia, 2011; Levinton, 2005; Luiz, 2005; Moreyra, 2012; Nahuelquir, 2012; Normando, 2013; Peña, 2012; Tardieu, 2005; Tiapa, 2007). Así mismo, la perspectiva de la antropología histórica y la etnohistoria ha abordado la dinámica de las interacciones que se establecieron entre grupos de personas indígenas, africanas, blancas y mestizas (Guzmán, 2010; Flagler, 2008; Rodríguez, 2012; Villar, 2007). En Colombia los análisis antropológicos e históricos han aportado en gran medida a la comprensión de las relaciones entre la población indígena, afrodescendiente y mestiza (Almario, 2009; Losonczy, 2006; Meza, 2003; Saether, 2005; Polo, 2012).

Amodio (1999) se interesa por la manera en que los pueblos indígenas del sistema interétnico del Orinoco transformaron su identidad para adaptarla a las condiciones mutables de las relaciones inter e intra-étnicas, en especial, bajo la presión de otros grupos regionales indígenas y europeos-. Para ello, analiza el caso de los indígenas caribes/kari'ña desde el periodo colonial hasta el periodo republicano. Afirma que las transformaciones identitarias y culturales se producen en el contacto con otros grupos étnicos y culturas. Así, por ejemplo, las alianzas de los caribes con los holandeses les permitió resistir a las imposiciones españolas casi hasta el final del periodo colonial.

Tardieu (2005) analiza la presencia de la población negra en los Andes y las formas de relacionamiento con los españoles y las comunidades indígenas en lo que actualmente es el 
territorio peruano. Presenta la complejidad de estas relaciones donde las víctimas de la colonización española también ejercieron en ocasiones como victimarios. De esta manera, el autor presenta varios matices de las relaciones interétnicas y el mestizaje en los Andes peruanos. Se establecieron matrimonios permitidos y alianzas políticas estratégicas entre indios y españoles y se presentaron casos de participación de población negra en batallas contra población indígena. Resalta el fracaso del sistema colonial de jerarquización social con el surgimiento de nuevas castas producto del mestizaje. En el caso de Esmeraldas, el surgimiento del "zambo" contribuyó a una alianza entre indios y cimarrones para el fortalecimiento de una zona que fue de difícil acceso y de tardía dominación española.

Peña (2009) analiza los diferentes mecanismos que utilizó la corona española para el traslado de esclavos, la inserción de los africanos en la sociedad colonial y su interrelación con la población indígena nativa en Chiapas y Guatemala. Su análisis histórico le permite describir los procesos de intercambio cultural reflejados en las tradiciones chiapanecas. Si bien no se profundiza en estas relaciones debido a la falta de registros, resalta los intercambios culturales que escaparon del marco de la imposición del sistema de castas que imponía la Corona española. Es decir, los indígenas y negros se aliaron huyendo de las imposiciones españolas y crearon relaciones de intercambio cultural y de mestizaje que definen características de lo que hoy son las tradiciones culturales y sociales en Chiapas.

Petruccelli (2003) investiga la reinserción ocupacional de la población esclava y su ubicación en un nuevo orden social después de la abolición de la esclavitud en dos regiones de Río de Janeiro a fines del siglo XIX. Realiza un abordaje comparativo entre las poblaciones de Vassouras y São Gonçal ya que muestran dinámicas poblaciones diferentes, producto de condiciones económicas que contribuyeron al mestizaje y organización de la población. Con este trabajo comparativo, el autor afirma que fue determinante la organización económica de los grupos para permitir sociedades más libres o cerradas. En el caso de Vassouras, la posesión de la tierra y la fuerte estructura socio-racial fomentó la discriminación racial y el desplazamiento a otras zonas de la población negra. En São Gonçal, debido a la proliferación de pequeños propietarios que accedieron a tierras, el mestizaje fue ligeramente aceptado. El acceso a la tierra contribuyó a la movilidad social y a un mayor relacionamiento entre blancos, pardos y negros en un sistema que exigía la segregación de la población.

Ibarra (2002) examina las formas y los mecanismos que los mestizos utilizaron para construirse como sujetos legítimos y litigantes en la ciudad de Quito en el siglo XVIII. La autora da cuenta del proceso de mestizaje como un hecho que demostraba la existencia de cierto tipo de fragmentación social y como un proceso continuo, sostenido y creciente. De este modo, concibe el mestizaje como un recurso y una estrategia que amplió su margen de acción hacia la construcción de un reconocimiento social y cultural.

Polo (2012) analiza una de las dimensiones clave de las relaciones interétnicas en el proceso de mestizaje en la provincia de Riocha: el poder y los simultáneos liderazgos que surgieron en el siglo XVIII. Examina cómo el papel de los intermediarios étnicos y la estrategia de cargos por las autoridades españolas resultaron no ser tan beneficiosas para el poder colonial. Para este autor, el mestizaje se dio en los niveles cultural, biológico y político con una preponderancia de elementos indígenas porque la filiación matrilineal de la cultura Wayuu fue muy importante para mantener la estructura social indígena. Si bien las autoridades españolas aprovecharon el papel de los mestizos-indígenas para posicionar sus propios intereses, en la práctica funcionó medianamente porque estos intermediarios mestizos utilizaron sus cargos para viabilizar sus intereses propios y los de sus familias, sobre todo en prácticas económicas como el 
contrabando.

El análisis de las relaciones interétnicas también abarca la historia contemporánea en escenarios con presencia de distintos grupos sociales en los que el mestizaje forma parte del discurso oficial y en los que las relaciones están atravesadas por el racismo. A partir del análisis de un proyecto de desarrollo en una comunidad maya del estado de Yucatán, México, y los discursos de sus promotores, Iturriaga y Rodríguez (2015) sostienen que la idea de desarrollo es un aura que enmascara una serie de relaciones de poder en los que se expresa, ejecuta y promueve un discurso en el cual el racismo se hace presente. Los autores afirman que el discurso que promueve la idea de "desarrollo" posibilita el control y manejo de personas y espacios, y en el caso estudiado, las relaciones interétnicas están entretejidas con las relaciones de clase cargadas de estereotipos y prejuicios raciales de parte de los promotores de desarrollo hacia la población indígena.

Ahora bien, la categoría de mestizaje no sólo ha sido usada para comprender ciertos contextos históricos y actuales, sino analizada en su potencial como concepto. A partir de la lectura de la producción académica de la tradición de la Escuela de Chicago, Cunin (2002) cuestiona conceptos como raza, mestizaje, hombre marginal y blanqueamiento para referirse a la manera cómo se analiza el mestizaje en América Latina y el Caribe. La autora parte del supuesto que el mestizaje, gracias a las imprecisiones y ambigüedades de quienes lo han estudiado, es un objeto pertinente y una herramienta heurística del análisis sociológico y antropológico que permite reflexionar sobre la construcción de las categorías sociales y científicas. Para la autora, no puede lograrse un análisis del mestizaje en términos de heterogeneidad o de homogeneidad sino que debe atenderse a su intermedio. Llama la atención sobre la idea de pensar el grupo racial como una condición previa a los grupos étnicos inscritos en la realidad social, pues pensar de esta forma es creer que toda la sociedad esta naturalmente formada por estos grupos.

Desde una perspectiva comparativa, Wade (2003) propone referirse a varios procesos de mestizaje y no solo a "el mestizaje". Para entender por qué esta idea tiene tanto arraigo en las naciones latinoamericanas se requiere ir más allá de entender el mestizaje como un proceso de exclusión disfrazada. Señala que hay diferentes procesos e ideas de lo que es mezcla, hay diferentes mestizajes, además, existen procesos de inclusión y exclusión a partir del mestizaje. Para el autor el mestizaje es múltiple y tiene muchos significados, está hecho de diferentes elementos y procesos que pueden manifestarse en el cuerpo, el ámbito familiar y la nación. En este sentido, el mestizaje es lo que marca la situación latinoamericana como sui generis y es muy distinta a la situación en Estados Unidos. Lo que marca la diferencia es el juego ambivalente y ambiguo entre la inclusión y la exclusión. Así mismo, el mestizaje y sus componentes están sujetos a las jerarquías de poder político y económico y a las jerarquías del racismo.

Desde una perspectiva crítica y feminista de un estudio en Chile, Salinas (2012) el mestizaje ha sido comprendido y enseñado desde un punto de vista: colonial y patriarcal. De este modo, el mestizo es una negatividad histórica, o una reiteración permanente de la violencia del "padre". En el proceso de la mezcla de las culturas, la participación activa y protagónica de las mujeres no occidentales concedió al proceso interétnico una dinámica más vital y creadora, distante de las representaciones oficiales del occidente colonial. Las mujeres indígenas, las mujeres moriscas y las mujeres africanas instalaron una forma de vivir donde la experiencia amorosa pasó a ser una experiencia fundante de la convivencia humana.

Los trabajos referenciados dan cuenta del mestizaje a partir de las relaciones interétnicas, visibilizan las transformaciones de la identidad étnica producto de las relaciones entre distintos grupos sociales y lo muestran como un proceso particular y localizado. El mestizaje funciona 
como una categoría de análisis que explica el curso de las relaciones interétnicas, pero también como el producto de relaciones estratégicas entre grupos étnicos para resistir, desestabilizar o negociar con los grupos sociales hegemónicos. Desde esta lógica, el mestizaje ha sido analizado como un mecanismo de movilidad e inserción en un sistema social de castas o de clases sociales permitiendo que las alianzas heterógamas viabilizaran la movilidad social. Entre tales alianzas, las relaciones maritales son una línea de análisis interesante que se aproxima a escenarios más privados y no solo colectivos.

\section{Relaciones maritales}

Varios de los estudios analizados abordan las relaciones interétnicas en relación con las uniones afectivas entre miembros de distintos grupos étnicos, raciales y culturales. De este modo, encontramos análisis de las relaciones maritales, las relaciones endogámicas y exogámicas, el carácter legítimo e ilegítimo de las uniones, y el resultado de las uniones: la familia y el parentesco. En el marco de estos análisis, las relaciones interétnicas son entendidas como las relaciones producto de la convivencia cotidiana entre diversas categorías socio-raciales: indígena, mestizo, mulato, español, ladino y esclavo.

Las uniones y matrimonios han sido analizados históricamente en un esfuerzo por comprender los procesos de mestizaje que se llevaron a cabo durante el periodo de colonización en América. Dueñas (1997a) examina algunos aspectos de la cultura popular que ponen en evidencia los altos índices de concubinato: la filiación ilegítima de la gran mayoría de niños nacidos a finales del siglo XVIII y la proliferación de la modalidad de hogares con jefatura femenina en Santafé, Nueva Granada. Afirma que durante este periodo la diversidad de uniones era lo usual y buena parte de la población no se acogía al rito católico del matrimonio impuesto por la Corona española. En otro estudio, Dueñas (1997b) da cuenta de cómo el concubinato, la ilegitimidad y el abandono de niños representaron una ruptura a las normas de la sociedad colonial a pesar de las imposiciones que fomentaban la desigualdad racial y de género de las élites blancas de la sociedad santafereña producto de dinámicas de mestizaje. La autora analiza la ilegitimidad, esto es, las uniones no permitidas dentro de los estándares de la iglesia y los hijos fuera de matrimonios católicos, a partir de las variables de género y de raza y las condiciones de posición social.

Saether (2005) indaga cómo las relaciones sociales en Cartagena y Santa Marta durante el siglo XIX dependieron de la posición social y la alineación política en la sociedad de castas basada en la separación social, cultural y política entre indígenas y españoles. El autor analiza las uniones ilegítimas fuera del marco matrimonial y que estaban prohibidas por las autoridades eclesiales. En el caso de los indígenas, examina la manera en que las relaciones maritales fueron utilizadas como estrategia para establecer relaciones con la sociedad colonial. Finalmente, en el caso de los esclavos africanos, explica que no se casaban tanto como en otros grupos, por resistencia a las instituciones españolas o porque no los dejaban. Resaltamos el aporte de este autor en tanto que hace un llamado al análisis de las fuentes de información que se basan en registros de autoridades pues han sido determinantes para la transmisión de la información desde una jerarquía social, en este caso, la iglesia católica. Al cuestionar la perspetiva histórica de las fuentes provenientes de una élite blanca, el autor superó la mirada unidireccional de los trabajos históricos y logró analizar la manera en que operaban las relaciones sociales en cada grupo social 
en la sociedad de castas.

Moreyra (2012) realiza un análisis histórico de dos estudios de caso de matrimonios entre personas de distinta extracción socio-étnica -españoles y pardas esclavas casadas por la Iglesiaen Córdoba, Argentina, a partir de una lectura sociocultural de la vida cotidiana y la cultura material. La autora aborda el mestizaje y los matrimonios interétnicos en la Córdoba colonial y problematiza la vida cotidiana recuperando la experiencia del sujeto. Considera que los matrimonios entre personas pertenecientes a distintos grupos étnicos generaban situaciones de movilidad social (ascendente y descendente).

Guzmán (2010) también explora el periodo colonial tardío en el Valle de Catamarca, Argentina, para dar cuenta de la presencia y agencia de la población negra en una zona donde comúnmente se desconoce su historia, particularmente de las mujeres negras esclavas y madres solteras, que transformaron las estructuras a través diversas estrategias, como las relaciones erótico-afectivas. El matrimonio otorgaba un estatus de legitimidad e integración en la sociedad de la época. Sin embargo, el matrimonio no fue un determinante para la procreación y el establecimiento de uniones sentimentales. El matrimonio con población libre fue una estrategia de los esclavos para conseguir su libertad. Esta fue una práctica extendida debido al aumento de mujeres que establecían uniones con libres, por lo que las autoridades eclesiales debieron permitir la libertad de los hijos producto de estas uniones. Así, la autora resalta la agencia de las mujeres negras para obtener algunos beneficios en una sociedad segregacionista.

Acuña (2012) analiza la dinámica del proceso de mestizaje en las poblaciones de Aserrí, Curridabat, Orosí, Tres Ríos y Escazú, provincia de Costa Rica, entre los años de 1750-1825. La autora señala que en poblaciones como Orosí, Aserrí y Curridabat existe una tendencia a uniones endogámicas en las poblaciones donde hay más presencia indígena, lo que permitió el crecimiento de su población. Afirma que los matrimonios con indígenas se dieron en muchas ocasiones para aprovechar los beneficios de tierras y protección que éstos tenían. La población mulata tuvo una tendencia mayor a la exogamia con indígenas y otras poblaciones mestizas dado los beneficios de estas relaciones y su condición precaria como esclavos. Así mismo, evidencia una mayor tendencia a uniones exogámicas en poblaciones como Tres Ríos producto de un número menor de indígenas y la falta de resistencia por mantener las tradiciones.

A través del análisis documental de los registros matrimoniales, Velázquez (2014) analiza el mestizaje y las relaciones interétnicas en la ciudad de Guanajuato en 1778. El mestizaje es un producto de la interrelación de los grupos étnico-sociales que puede ser analizado a partir de indicadores tales como la legitimidad e ilegitimidad, la soltería o viudez, la procedencia de los contrayentes o la edad del matrimonio. En este trabajo, las relaciones interétnicas son entendidas como la fusión de diferentes grupos étnicos y de diversas culturas cuyo producto sería el mestizaje. La convivencia entre distintos grupos étnicos y sociales engendraba relaciones de todo tipo: parentesco (matrimonio, compadrazgo, padrinazgo) y de amistad.

En un contexto más reciente, García (2010) analiza los discursos y percepciones de los diferentes grupos culturales presentes en el territorio de San Andrés, Colombia, para examinar la situación de los nuevos sujetos híbridos de las islas con el fin de identificar la pertenencia a la etnicidad raizal. Estos sujetos híbridos denominados Half\&Half o FiftyFifties son el producto de uniones y matrimonios entre la población raizal y la población del continente colombiano que migró a las islas. La autora problematiza los escenarios en donde los Half\&Half o Fifty-Fifties han sido excluidos convirtiéndose en sujetos invisibles al interior de la etnia raizal, así como los escenarios en donde Half\&Half o FiftyFifties se han integrado en el territorio, dando paso al reconocimiento y formación de una sociedad insular basada en la interculturalidad. 
Vale la pena resaltar la atención que estos estudios prestan a los roles de las mujeres en las relaciones interétnicas. Sin embargo, aunque trabajos como el de Dueñas (1997a, 1997b) avanzan en una perspectiva de género en los análisis históricos, las relaciones interétnicas son abordas desde las interacciones de sujetos colectivos sin detenerse en el detalle de las experiencias particulares y diversas de las personas, relaciones profundamente jerarquizadas cuando variables como el sexo, la raza, la adscripción étnica afectan de manera diferenciada a hombres y mujeres. Las uniones maritales entre personas de distintos grupos étnicos son un escenario que también toca la dimensión íntima que por serlo no deja de estar atravesada por relaciones de poder y discriminación.

\section{Discriminación}

Las relaciones entre grupos étnicos y sociales diversos pueden originar situaciones de desigualdad, marginación y discriminación. Desde una perspectiva antropológica, encontramos trabajos en los que las relaciones interétnicas se analizan a partir de la construcción de representaciones, imaginarios o estereotipos frente a los otros (Murray, 2010; Ortega, Nissen \& Castillo, 2006; Benedetti, 2012; García, 2010; Bartolomé, 2014). Los procesos de nominación y adscripción en el contacto interétnico conllevan estigmatizaciones, prejuicios y señalamientos (Liberona, 2015; Benedetti, 2012) que devienen en exclusión. Algunos estudios definen las relaciones interétnicas a partir de las resistencias, transgresiones o rupturas con las instancias de dominación (Albadán, 2012). La identidad se vuelve un instrumento de visibilización, reivindicación y estrategia política para la inserción de determinados grupos en la arena política y estatal (Gonzáles, 2006; Pisano, 2014; Sámano, 2005; Soler, 2013; Tiapa, 2011) o para el diálogo con las instancias de poder (Ramos, 2015).

Oehmiche (2010) analiza la violencia y el racismo en el marco de las relaciones indomestizas en México. Parte del uso del concepto de "violencia simbólica" sobre la cual descansa las representaciones que tienden a criminalizar a los indígenas. La violencia simbólica permite reproducir y perpetuar las relaciones de dominación, está enraizada en la cultura y se ejerce con la participación activa y el consentimiento de los dominados para perpetuar su propia dominación. A partir del uso del concepto de violencia simbólica, las relaciones interétnicas (relaciones entre indígenas y mestizos) son comprendidas como relaciones asimétricas donde la violencia se expresa con mayor o menor crueldad e intensidad dependiendo del contexto de interacción y de los intereses en juego.

El trabajo de Castillo (2003) analiza la manera en que los estereotipos orientan las relaciones entre diversos grupos étnicos. La autora examina la forma en que mixtecos, mestizos y afro mestizos en Pinacoteca Nacional, Oaxaca, se relacionan entre sí de manera desigual y establecen relaciones de dominación y subordinación recreando así estereotipos. Estos grupos se ubican de manera diferencial dentro de la sociedad estableciendo relaciones de poder y subordinación. En esta medida, las relaciones interétnicas las define como un conjunto de relaciones "estrictamente elaboradas y sancionadas (...) que en gran medida se configuran por los estereotipos que integran su identidad étnica" (p.268).

A partir de un enfoque antropológico, Bartolomé (2014) intenta demostrar que las culturas indígenas de Oaxaca, México, no sólo están crónicamente empobrecidas, sino que también son mal conocidas e imaginadas, ya que están sometidas a la proyección de los prejuicios y fantasías 
de la sociedad no indígena, que convive con ellas en el marco de un proceso interétnico de varios siglos de duración. El autor propone una crítica al aparente discurso de un diálogo intercultural cuando, en realidad, se trata de la imposición de lenguajes y formas de comunicación hegemónicas. Las relaciones interculturales estarían dadas por los procesos de comunicación en los que participan individuos o grupos pertenecientes a diferentes culturas y en los que la diferencia cultural proporciona distintas formas de percepción y de conducta.

Pinkus, Pacheco y Lugo (2011) también reflexionan, desde una perspectiva antropológica y sociológica, sobre las relaciones que establecen 47 comunidades rurales del municipio de Mérida, en México, con la población de la cabecera municipal. Los autores afirman que la pobreza y la marginación de los pueblos indígenas mayas yucatecos son el producto del contexto histórico y de las transformaciones estructurales que el estado ha impuesto sobre todo a partir del impulso de la política económica neoliberal. En este contexto, las relaciones interétnicas son relaciones que se dan entre grupos que se identifican como mayas yucatecos y la población mestiza de la cabecera municipal, relaciones que están marcadas por vínculos desiguales e inequitativos que los grupos dominantes han impuesto. De este modo, los grupos hegemónicos se distinguen de los grupos sociales que consideran ajenos a ellos y legitiman su posición de dominio.

Gómez (2013) describe la visibilización de los pueblos originarios en la provincia del Chaco y explora algunos elementos del proceso de mercantilización de la cultura de estos pueblos en el marco de las actividades turísticas. Esta situación se da en el marco de políticas socioeconómicas excluyentes y de relaciones interétnicas desiguales y discriminatorias a partir de un conflicto de clase social entre indígenas y mestizos. En las relaciones interétnicas intervienen el Estado, los grupos indígenas y otros actores sociales, ubicando a los segundos en una posición de desigualdad social y explotación económica. La autora introduce al análisis la variable de clase social entendiéndola como una contradicción de las relaciones interétnicas: el conflicto de clase. Esta contradicción es ocultada a través de las representaciones y estigmas que recaen sobre la imagen de los pueblos indígenas e invisibilizan las condiciones materiales reales.

Por su parte, Benedetti (2012), investiga la producción artesanal chané de Campo Durán, Argentina, y enfatiza en la dinámica de relaciones interétnicas que atraviesa a la misma. Desde una perspectiva cualitativa de la antropología social y su trabajo de campo, la autora centra el análisis en la articulación de las artesanías en un "entramado de relaciones interétnicas configurado históricamente, donde se construyen las identificaciones de los sujetos sociales" (p.23). De este modo, examina la artesanía a partir de su inserción en el sistema económico y sostiene que los imaginarios en torno a dicha actividad sólo pueden ser comprendidos desde las interacciones y fricciones entre los indígenas y los criollos. Los estigmas clasifican y califican a los pueblos originarios lo que lleva las construcciones identitarias a un campo de desvalorizaciones despectivas que reproducen desigualdades y diferencias sociales.

El trabajo de Hoogbergen y Krujit (2004) se adentra en los yacimientos de oro de Surinam donde se encuentran pueblos cimarrones y donde a partir de los años 90 se ha originado un movimiento migratorio de garimpeiros brasileños modificando el escenario demográfico de la región. Los autores describen la dinámica de los yacimientos de oro y sugieren que la rivalidad potencial que puede haber entre ambos grupos no es tan tensa como se supone. En este caso, las tensiones étnicas que han sido canalizadas a través del rol de partidos políticos que representan los diversos grupos.

Gunderman, González y Durston (2014) abordan las relaciones interétnicas enfocándose en los actores sociales, su subjetividad y las prácticas que despliegan en los espacios de vinculación interétnica. Para esto se enfocan en el caso aymara chileno el cual guarda una 
relación estrecha con la política de acción afirmativa iniciada por las agencias públicas desde la década de 1990. En el marco del estudio, el elemento definitorio de estas relaciones es el sentido de pertenencia, adscripción y participación en un pueblo indígena, de personas y grupos en relación con otros que mantienen y hacen relevantes otros sentidos de pertenencia colectiva, étnicos o no.

Con una mirada crítica y contextual, los trabajos referenciados arrojan luces sobre las relaciones interétnicas a partir del análisis de situaciones discriminación en contextos sociales más recientes. Procesos y dinámicas migratorias, políticas, económicas y socioculturales de los siglos XX y XXI modifican el carácter de las relaciones entre grupos poblaciones diversos y demuestran que las relaciones interétnicas no se dan en contextos armoniosos y de manera horizontal. Pero los análisis continúan centrándose en sujetos colectivos y las situaciones de discriminación operan de manera desigual en la cotidianidad de las personas, valdría la pena ahondar aún más en estas experiencias cotidianas que no necesariamente se desenvuelven en terrenos políticos o económicos.

\section{Reflexiones finales}

El carácter relacional de lo interétnico atraviesa todos estos estudios y supone la comprensión de las dinámicas de grupos con suscripciones étnicas diversas que interactúan y ponen en juego sus intereses configurando relaciones de conflicto, tensión, contacto, diálogo, intercambio, vínculo, alianza, integración y negociación. Además de cuestionar las posiciones asimétricas, la dimensión del poder en el análisis de las relaciones interétnicas permite pensar en espacios de frontera como campos de fuerzas donde las diferencias no solo se imponen sino también se resisten y se negocian.

A partir de la revisión de los documentos académicos referenciados, observamos que son muy pocas las posturas que se enfocan en la revisión del concepto de relaciones interétnicas y, en cambio, en la mayoría de los estudios este concepto es aplicado para referirse de manera general a las relaciones entre las minorías étnicas y la población hegemónica blanco-mestiza. Al mismo tiempo, son escasos los análisis que tienen en cuenta las experiencias particulares y situadas de las personas atendiendo a las relaciones de género. Las relaciones superan la esfera abstracta e intangible de lo social y se encarnan en los cuerpos de individuos con problemas cotidianos. En esta medida, valdría la pena preguntarse por la condición de mujeres y hombres que activan y viven lo étnico social y corporalmente de manera diversa, pues el cuerpo étnico también es un cuerpo sexuado sometido a poderes que lo definen y lo normalizan.

De igual modo, es necesario superar la visión según la cual existen las relaciones interétnicas per se en un contexto social determinado. La comprensión de las relaciones entre grupos étnicos debe incluir una mirada sistémica, histórica y crítica como varios de los estudios lo proponen. En este sentido, resulta sugerente referirse a lo étnico como una construcción social cuyo carácter identitario de sujetos individuales y colectivos puede ser estratégico y activado según las necesidades históricas a partir de la agencia y no solo de la imposición. Esta mirada no solo debe aplicarse al terreno de lo político, sino que es pertinente que se amplíe a ámbitos como el cultural y personal.

Más allá del determinante social, podemos también comprender la situación particular de los sujetos étnicos a partir de las prácticas, inscripciones y posicionamientos de etnicidad que 
despliegan en los espacios de vinculación o relación interétnica. En este sentido, lo interétnico es estratégico para la inserción en ciertos espacios y puede activarse con referencia a un reconocimiento colectivo, y en esta medida, tiene un carácter político y activo que supera la idea de lo étnico como subordinado. En este sentido, podemos analizar las relaciones interétnicas en espacios de poder, pero también de inteligibilidad mutua y diálogo a partir de las diferencias, un intersticio donde lo étnico puede, incluso, estar ausente.

\section{Bibliografía}

Acuña, M. (2012). “Mestizaje y relaciones interétnicas en Aserrí, Curridabat, Orosi, Tres Ríos y Escazú 1750-1825”. Cuadernos Intercambio, Año 10, Vol. 10(11), 79-104.

Albadán, C. (2012). “Celebraciones en San Andrés: participación, convivencia e inserción raizal". Revista Cuadernos del Caribe: Problemas agropecuarios en San Andrés isla: análisis transdisciplinarios, (7), 34-45.

Almario, O. (2009). “De lo regional a lo local en el pacifico sur colombiano, 1780-1930”. HiSTOReLo, Vol. 1(1), 76-123.

Amodio, E. (1999). "Los caníbales mutantes. Etapas de la transformación étnica de los Caribes durante la época colonial'. Boletín americanista, $\mathrm{N}^{\circ}$ 49, 9-29.

Bari, M. (2002). "La cuestión étnica: Aproximación a los conceptos de grupo étnico, identidad étnica, etnicidad y relaciones interétnicas". Cuadernos de Antropología Social, No 16, 149-163.

Bartolomé, M. (2014). “Las identidades imaginadas en Oaxaca. Algunos problemas del diálogo intercultural en una sociedad plural'. Cuicuilco, (60), 83-108.

Benedetti, C. (2012). Diferencias y desigualdades: reflexiones sobre identidad étnica y producción artesanal chané destinada a la comercialización. Alteridades, 22(43), 21-33.

Biord, H. (2005). "Sistemas interétnicos regionales: el Orinoco y la costa noreste de la actual Venezuela en los siglos XVI, XVII y XVIII'. SERIE: Cuadernos del GIECAL, No 2 85-120.

Bossert, F. \& Siffredi, A. (2011). "Las relaciones interétnicas en el Pilcomayo medio: la guerra indigena y sus transformaciones (1882-1938)”. Población \& Sociedad, Vol. 18(1), 3-47.

Buscaglia, S. (2011). "La representación de las relaciones interétnicas en el discurso de Antonio Viedma (Patagonia Meridional, siglo xviii)”. Magallania, Vol. 39(2), 15-35.

Cabrera, S. (2009). "Relaciones interétnicas y cuestiones limítrofes en el espacio fronterizo de la norpatagonia. Rupturas y continuidades durante el proceso de conformación de los Estado-nación argentino y chileno". Revista TEFROS, Vol. 7(1-2), 1-19.

Calderón, F. (2016). "Repensando la frontera africana de Castilla: ¿Una frontera interétnica?”. Frontera Norte, Vol. 28(55), 5-31.

Casali, R. (2014). "Salud en los extremos. Escenario epidemiológico y sanitario en Tierra del Fuego, 1890-1930”. Rev. Cienc. Salud, 12(2), 271-88.

Castillo, A. (2003). "Los estereotipos y las relaciones interétnicas en la Costa Chica Oaxaqueña". Revista Mexicana de Ciencias Políticas y Sociales No. 46: 267-90. 
Córdoba, L. \& Villar, D. (2010). "Relaciones Interétnicas, etnonimia y espacialidad: el caso de los panos meridionales". Boletín Americanista, Año LX.1(60), 33-49.

Cunin, E. (2002). "La competencia mestiza. Chicago bajo el trópico o las virtudes heurísticas del mestizaje". Revista Colombiana de Antropología, Vol. 38, 11-44.

Dueñas, G. (1997a). "Las mestizas y el concubinato en el patriarcal Santafé de Bogotá, siglo XVIII'. En otras palabras... (3), 49-58.

Dueñas, G. (1997b). Los hijos del pecado: ilegitimidad y vida familiar en la Santafé De Bogotá Colonial. Bogotá: Universidad Nacional de Colombia.

Fabbricatore, E. (2011). Las formas de la convivencia interétnica. Una indagación cualitativa. Prismasocial, (6), 6-22.

Flagler, E. (2008). "Relaciones interétnicas: los indios zuñi de Nuevo México y los españoles". Revista Española de Antropología Americana, Vol. 38 (2), 57-73.

Foerster, R. \& Vezub, J. (2011). “Malón, ración y nación en Las Pampas: el factor Juan Manual de Rosas (1820-1880)”. Historia, Vol. II (44), 259-286.

Galarza, A. (2012). "Relaciones interétnicas y comercio en la frontera sur rioplatense. Partidas indigenas y transacciones comerciales en la guardia de Chascomús (1780-1809)”. Fronteras de la Historia, Vol. 17(2), 102-128.

García, S. (2010). Los "Half \& Halfo Fifty Fifties de San Andrés. Los Actores Invisibles de la Raizalidad. Universidad Nacional de Colombia, Sede Caribe.

Gómez, S. (2013). "Pueblos originarios y turismo en la provincia del Chaco: construcción de "lo indígena" y mercantilización de la cultura". Cuadernos de Antropología, N 9.

González, M. (2006). "Relaciones interétnicas en la argentina del siglo XX. La nueva utopía entre la realidad y la ideología". Revista TEFROS, Vol. 4 № 1.

Grebe, M. (2012). "Ciudadanía, constituciones y relaciones interétnicas en la Sierra Ecuatoriana (1812-1830)”. Procesos Revista Ecuatoriana de Historia, 36, II semestre, 73110.

Gunderman, H., González, H. \& Durston, J. (2014). "Relaciones sociales y etnicidad en el espacio Aymara chileno". Revista de Antropología Chilena, Vol. 46(3), 397-421.

Guzmán, F. (2010). Los claroscuros del mestizaje: negros, indios y castas en la Catamarca Colonial. Córdoba: Encuentro grupo editor.

Hernández, R. (2003). "Caciques, jesuitas y chamanes en la frontera sur de Buenos Aires (17401753) ". Anuario de Estudios Americanos, Tomo LX, I, 77-108.

Hoogbergen, W. \& Kruijt, D. (2004). "Gold, garimpeiros and maroons: brazilian migrants and ethnic relationships in post-war Surinam". Caribbean Studies, Vol. 32(2), 3-44.

Ibarra, A. (2002). Estrategias Del Mestizaje: Quito a Finales Del Siglo XVIII. Quito: Ediciones Abya-Yala, Agencia Española de Cooperación Internacional, Pontificia Universidad Católica de Ecuador.

Iturriaga, E. \& Rodríguez, Y. (2015). "Racismo y desarrollo: el proyecto turístico uh najil en Ek Balam, Yucatán”. Península, Vol. X(2), 49-70.

Lenaerts, M. (2011). “Etnobotánica ashéninka, entre la "tradición” y la "modernidad”: ¿qué significa "conocer las plantas"?”. Mundo Amazónico 2, 67-94.

Levinton, N. (2005). "Las estancias de Nuestra Señora de los Reyes de Yapeyú: tenencia de la tierra por uso cotidiano, acuerdo interétnico y derecho natural (Misiones jesuiticas del Paraguay)”. Revista Complutense de Historia de América, Vol. 31, 33-51. 
Liberona, N. (2015). "De las fronteras geopolíticas a las fronteras sociales. La migración boliviana a través de la prensa de Tarapacá (1990-2007)”. Estudios Fronterizos, Vol. 16(32), 41-74.

Losonczy, A., \& Rubiano, J. (2013). “La política por los espíritus: escenarios multiculturales en 'zonas de contacto' (Valle de Cauca, Colombia)”. Religião \& Sociedade, 33(1), 11-29.

Losonczy, A. (2006). Pasado y presente de los negro-colombianos del chocó y el campo interétnico: representaciones e intercambios entre los negros y los Emberá. En La trama interétnica: ritual, sociedad y figuras del intercambio entre los grupos negros y Emberá del Chocó. Bogotá: Instituto Colombiano de Antropología e Historia e Instituto Francés de Estudios Andinos.

Luiz, M. (2005). "Re-pensando el orden colonial: los intercambios hispano-indígenas en el fuerte del río Negro". Mundo Agrario, Vol. 5(10).

Meza, A. (2003). Trayectorias de los afrodescendientes en el comercio callejero de Bogotá. Revista Colombiana de Antropología. Vol. 39, 71-104.

Moreyra, C. (2012). "Mestizaje, vida cotidiana y cultura material una mirada sociocultural a dos matrimonios interétnico en la ciudad de Córdoba, Siglo XVIII”. Diálogos Revista Electrónica de Historia, vol. 13(2), 92-111.

Murray, G. (2010). Dominican-Haitian racial and ethnic perceptions and sentiments: mutual adaptation, mutual tensions, mutual anxieties. Santo Domingo: MIF Fomin y PADF.

Nahuelquir, F. (2012). "Políticas de territorialización de indígenas en Chubut: provincialización de las relaciones interétnicas a través del acceso a la tierra". Revista Latinoamericana de Metodología de las Ciencias Sociales, julio-diciembre, Vol. 2(2), 72-108.

Normando, E. (2013). “'Esclavos españoles, indios y negros': notas para el estudio de las relaciones interétnicas en las cofradías religiosas del norte del Virreinato del Río de la Plata". Boletim do Museu Paraense Emílio Goeldi. Ciências Humanas, 8(2), 449-458.

Oehmiche, C. (2010). "Violencia en las relaciones interétnicas y racismo en la Ciudad De México". Cultura y representaciones sociales, Vol. 1(2), 91-117.

Ortega, M., Nissen, M. \& Castillo, M. (2006). Diversidad, identidades y relaciones interétnicas en Nicaragua. La costa Caribe y el Pacífico: conocimientos y percepciones interregionales. Cuadernos de investigación, Vol. 22.

Peña, J. (2009). "Relaciones entre africanos e indígenas en Chiapas y Guatemala". Estudios de Cultura Maya, $N^{\circ} 34,167-80$.

Petruccelli, J.L. (2003). "Grupos sociales y mestizaje en el Estado de Río de Janeiro a fines del siglo XIX”. Contribuciones desde Coatepec, № 5, 99-114.

Pinkus, M., Pacheco \& J., Lugo, J. (2011). "Las poblaciones rurales de Mérida y sus relaciones interétnicas con esta ciudad capital de la entidad yucateca de México". Revista Pueblos y Fronteras Digital, Vol. 6(12), 236-267.

Pisano, P. (2014). "Movilidad social e identidad "negra" en la segunda mitad del siglo XX". Anuario Colombiano de Historia Social y de la Cultura, 2014, Vol. 41(1), 179-199.

Polo, J. (2012). Mestizaje, intermediarios étnicos y liderazgo indígena. En Indígenas, poderes y mediaciones en la Guajira en la transición. De la Colonia a la República (1759-1850). Universidad Central de Venezuela.

Ramos, A. (2015). "Sobre malentendidos interétnicos". Universitas Humanística, № 80, juliodiciembre, 53-75. 
Ratto, S. (2005). “Caciques, autoridades fronterizas y lenguaraces: intermediarios culturales e interlocutores válidos en Buenos Aires (primera mitad del siglo XIX) ”. Mundo Agrario, Vol. 5(10).

Reyes, V. (2004). "Relaciones interétnicas en asentamientos del siglo XVI de la Precordillera Lacustre, IX y X Regiones: Análisis Cerámico Chungara”. Revista de Antropología Chilena, Vol. 36(1), 161-174.

Rodríguez, E. (2013). Choles, mayas y mestizos en el sur de Yucatán. Península, Vol. VIII(2), 6585.

Rodríguez, S. (2012). "Poblaciones blancas en el pacífico: historia y vigencia". Maguaré, $\mathrm{N}^{\circ} 15-$ $16,114-35$.

Rodríguez, L. \& Porraz, I. (2009). “Del barrio a la comunidad: religiosidad popular y relaciones de poder en el sureste de México". Maguaré, (23), 65-102.

Saether, S. (2005). Identidades e independencia en Santa Marta y Riohacha. Bogotá: Instituto Colombiano de Antropología e Historia.

Salinas, M. (2012). "Las mujeres indígenas, moriscas y africanas: los mestizajes y la representación de la sociabilidad amorosa en Chile". Chungara, Revista de Antropología Chilena, Vol. 44(2), 325-340.

Sámaro, M. (2005). “Identidad étnica y la relación de los pueblos indígenas con el estado mexicano". Ra Ximhai, Vol. 1(2), 239-260.

Schindler, H. y Faust, F. (2000). "Relaciones interétnicas de los curanderos en el suroccidente colombiano". Anthropológica, 18(18), 281-94.

Soler, S. (2013). "Entre negro oscuro y moreno claro: discursos e identidades étnicas en niños y niñas afrodescendientes en contexto escolar en Bogotá". Educar em Revista, (47), 111143.

Tardieu, J. (2005). “Relaciones interétnicas en la zona andina. Época Colonial”. Revista del CESLA, N ${ }^{\circ} 7,151-86$.

Tiapa, F. (2011). "Identidad, alteridad y relaciones interétnicas en las fronteras de la Modernidad. Una aproximación desde la teoría antropológica Fermentum". Revista Venezolana de Sociología y Antropología, Vol. 21(62), 384-414. . (2007). "Las relaciones interétnicas entre los warao de la frontera noroccidental del delta del Orinoco durante la época colonial'. Trocadero, (19), 215-228.

Valerezo, G. (2003). "Relaciones interétnicas en la Sierra Norte: la Diversa Evolución de Cayambe y Otavalo en el tránsito a la República". Procesos, Revista Ecuatoriana de Historia, (19), 23-48.

Velázquez, G. (2014). "Mestizaje y matrimonio en la ciudad de Guanajuato en 1778. Una aproximación a su estructura socio-demográfica". Tzintzun Revista de Estudios Históricos, $\mathrm{N}^{\circ}$ 60, 74-104.

Villar, D. (2007). "Religiones omnívoras: el chamanismo chané y las relaciones interétnicas". Anthropologica del Departamento de Ciencias Sociales, Vol. XXV(25), 157-170.

Wade, P. (2003). Repensando el mestizaje. Revista Colombiana de Antropología, Vol. 39, 27396.

(1993). Gente Negra, Nación Mestiza Dinámicas De Las Identidades Raciales en Colombia. Bogotá: Siglo del Hombre Editores, Ediciones Uniandes. 